CONCURSO ESTUdiANTIL DE DISEÑO

\title{
Tisekuae Xuta Xtaya: Ayuda a las personas sordas
}

\author{
Eduardo Armas García, Rafael Cano Martínez, \\ Víctor Hernández Vásquez, Luis C. Mota Carrera, \\ María del Rosario Peralta Calvo
}

Publicado: 31 Octubre 2018

\begin{abstract}
Resumen
Una gran parte de la población mundial presenta discapacidad auditiva, las consecuencias derivadas de esto, repercuten en aspectos importantes de su vida, como el desarrollo cognitivo y socioafectivo, la educación, la comunicación y la personalidad, por mencionar algunas. Tisekuae Xuta Xtaya, es una aplicación móvil diseñada para ayudar a los niños sordos en el contexto educativo y al mismo tiempo que aprenden el Lenguaje de Señas Mexicano (LSM). El objetivo de la aplicación es que el niño mejore su capacidad cognitiva ejercitando su memoria conceptual, de cara a que en un futuro tenga la habilidad de comunicarse sin problemas, coadyuvando en la mejora de la calidad de vida de las personas con discapacidad auditiva.
\end{abstract}

Palabras clave: Sordo; Diseño Centrado en el Usuario; Estudio Contextual, Memoria Conceptual, LSM.

\section{Introducción}

Las personas que presentan discapacidad auditiva se enfrentan a dificultades para el correcto aprendizaje y realización de diferentes actividades a lo largo de su vida. Hasta el año 2014, la Federación Mundial del Sordo estima que 70 millones de personas usan el lenguaje de señas de las 360 millones de personas que sufren pérdida auditiva, según la Organización Mundial de la Salud (OMS) [1]. Las causas de este mal son principalmente por dos: las congénitas y las adquiridas. La primera determina la pérdida de la escucha en los primeros momentos del nacimiento o poco después. La segunda causa ocurre a cualquier edad y sucede de diversas formas, como infecciones, uso de medicamentos de forma descontrolada, el envejecimiento, exposición a sonidos muy ele-vados o traumatismo craneoencefálicos o de los oídos [1]. De acuerdo con los resultados de la ENADID 2014, de los 119.9 millones de personas que habitan México, 6\% (7.2 millones) tienen discapacidad, de las cuales el $33.5 \%$ su problema es auditivo y el $18 \%$ su problema es para hablar o comunicarse [2]. Del 6\% de población con discapacidad a nivel nacional, en

Armas García E., Cano Martínez R., Hernández Vásquez V., Mota

Carrera LC., Peralta Calvo MR.

Universidad de la Cañada

Km. 1.7 Carretera Teotitlán-Nanahuatipán,

Teotitlán de Flores Magón, Oaxaca

Email: li_eduardoag@unca.edu.mx, li_rafaelcm@unca.edu.mx,

li_victorhv@unca.edu.mx, li_luismc@unca.edu.mx,

mperalta@unca.edu.mx
Oaxaca reside el $3.6 \%$, de las cuales el $36.2 \%$ y $19.4 \%$ tienen problema auditivo y problema para comunicarse, respectivamente [2].

\section{Problemática}

Tener una educación no consiste únicamente en aprender a leer y escribir, sino que constituye la base del desarrollo personal y social desde las primeras etapas de vida de las personas. A pesar de la importancia de la educación, en México esta población vulnerable difícilmente puede acceder a una educación especial ya que las instituciones públicas no están especializadas a tratar a niños con discapacidad auditiva y por ende, los modelos de aprendizaje y formación que habitualmente se imparten en las escuelas no son aptos para los niños sordos, en consecuencia la gran parte de su educación básica se realiza en lenguaje Español Mexicano y no en el Lenguaje de Señas Mexicano (LSM). De esta manera, el grado de comprensión y aprendizaje respecto a los otros estudiantes se torna inferior, esto se agrava con otras causas que trae consigo la discapacidad, como la dificultad en la comunicación, la socialización, los procesos cognitivos y la memoria. Además, lo mencionado repercute negativamente en el proceso académico y limita de forma importante al niño en la edad escolar. Poner especial atención en la educación de los niños sordos, les brindará un mejor desarrollo e integración con su entorno.

\section{Solución}

\subsection{Trabajo relacionado}

El desarrollo de recursos tecnológicos han coadyuvado a la enseñanza educativa para niños con diferentes discapacidades, a continuación se describen algunas que han apoyado a niños sordos. Multimedia Kimera (MK), su enfoque es el desarrollo de software educativo, cuenta con software para niños en preescolar y primaria. Así como distintos programas para distribuir textos de referencia como diccionarios o biografías. Los productos de MK han ayudado a acercar a los niños sordos al Español, y a estimular sus habilidades perceptivas y de motricidad, favoreciéndolos con una serie de juegos enfocados a la enseñanza de los conceptos básicos del Español. Sin embargo, a pesar de esto no cuenta con un pro-ducto diseñado exclusivamente para personas sordas en el que se enseñe el LSM. Cabe resaltar, que la interfaz gráfica con la que cuenta resulta atractiva para los niños y la implementación de un juego como medio de enseñanza. El uso de los distintos productos ofrecidos por Kimera ha sido uno de los primeros acercamientos tecnológicos entre el aula de clase y el mundo 
multimedia desde 1998 [3]. Hablando con Julis (HCJ), en su versión pedagógica y comunicativa, presenta una solución para el aprendizaje y la comunicación, enfocado al beneficio de personas con dificultades en el habla: personas con síndrome de Down, personas con autismo, personas con parálisis cerebral, solo por mencionar algunas. El usuario por medio de una interfaz gráfica puede seleccionar imágenes asociadas a palabras, acciones, objetos, lugares y personas, con las cuales se pueden formar frases y obtener el audio de éstas, utilizando una interfaz interactiva. Los grupos vulnerables a los que puede apoyar presentan diferentes necesidades y contexto, por lo que HCJ no cubre en específico a las personas sordas [4].

\subsection{Propuesta}

Considerando la importancia que tiene compartir el mismo código de comunicación en una comunidad de personas sordas, para el desarrollo personal y social, se propone la implementación de una aplicación móvil como una herramienta en el apoyo educativo en las primeras etapas de desarrollo escolar. Con esto los niños sordos podrán aprender el LSM, ejercitando su comunicación y aprendizaje por medio de representaciones gráficas como símbolos, imágenes, iconos, videos o animaciones a través de escenarios, para que los usuarios puedan identificar y familiarizar con mayor facilidad los elementos presentados. También, se apoyaría a la estimulación de la memoria conceptual para adquirir información y dar significado a las cosas.

Siguiendo las bases que ofrece la Guía de Deaf Child Worldwide, que consiste en la orientación para profesores, cuidadores, padres y otros familiares de niñas y niños sordos, con el fin de planificar, implementar y dar seguimiento de la enseñanza de la lengua de señas. Además de sugerir temas específicos que pueden ser abordados en ciertos niveles de educación escolar [5].

\section{Metodología UCD}

El Diseño Centrado en el Usuario (DCU) es un término amplio para describir los procesos de diseño en los que usuarios finales influyen de manera directa en el diseño del producto. Existen varias maneras en que los usuarios se involucran en este proceso, por ejemplo, se les consulta acerca de sus necesidades y se les involucra en momentos específicos durante el proceso de diseño, por lo general en la obtención de requisitos y pruebas de usabilidad [6]. La presente propuesta se enfoca en tres de los aspectos de esta metodología (estudio, diseño y evaluación), debido a la temática manejada. En la fase de estudio, se realizó un estudio contextual; en la fase de diseño, una versión prototipo basada en prototipos de baja fidelidad y en la fase de evaluación, se realizaron pruebas de mago de oz.

\subsection{Estudio}

Para conocer el contexto y necesidades donde interactúan las personas sordas, se realizó un estudio contextual en la comunidad de Teotitlán de Flores Magón los días 20, 21 y 22 de abril, el cuál consistió en pláticas y entrevistas in-situ con los tutores de las personas con discapacidad auditiva. Los temas de interés del estudio fue conocer la manera en que desempeñan las actividades cotidianas, la cercanía con la tecnología, si han recibido atención especializada por la discapacidad y el tipo de trato que han recibido en el entorno social y en instituciones públicas. Con el análisis de las respuestas e información brindadas por las familias en el estudio contextual, se detectó que: las personas con esta discapacidad, no se ven imposibilitados de realizar actividades cotidianas. Sin embargo, su principal limitante es la comunicación, lo cual impacta en el aspecto educativo. La mayoría de las familias de las personas con discapacidad auditiva cuentan con un dispositivo móvil, lo que indica que hay contacto con la tecnología. La mayoría realizan o realizaron sus estudios académicos en las instituciones de la población, con ciertas atenciones de los docentes, aunque poco especializadas, porque no tuvieron una preparación para dar atención a niños sordos, lo cual ha tenido repercusión en el proceso de enseñanza-aprendizaje de niveles avanzados. También, suele reflejarse cierta exclusión de los estudiantes con discapacidad auditiva en muchas actividades, al no recibir la misma atención que el resto de los alumnos en el salón de clases. Una minoría ha estudiado en instituciones con especialización en la discapacidad, pero estas se ubican en la capital del país. Como consecuencia a que el modelo de enseñanza de las instituciones de la población no está destinado a los niños sordos, dicha deficiencia en la atención no contribuye al desarrollo de su capacidad conceptual a una temprana edad, por lo que es apremiante considerar herramientas que permita apoyar en el proceso de enseñanza de los niños sordos. Se recurre a un storyboard para representar el escenario de un niño sordo en su contexto escolar. El storyboard se desarrolla en un salón de clases donde el profesor trata de incluir a un niño sordo dentro de las actividades del aula de clases, pero la atención no es la adecuada y se derivan complicaciones en el aprendizaje del niño sordo (Figura 1).

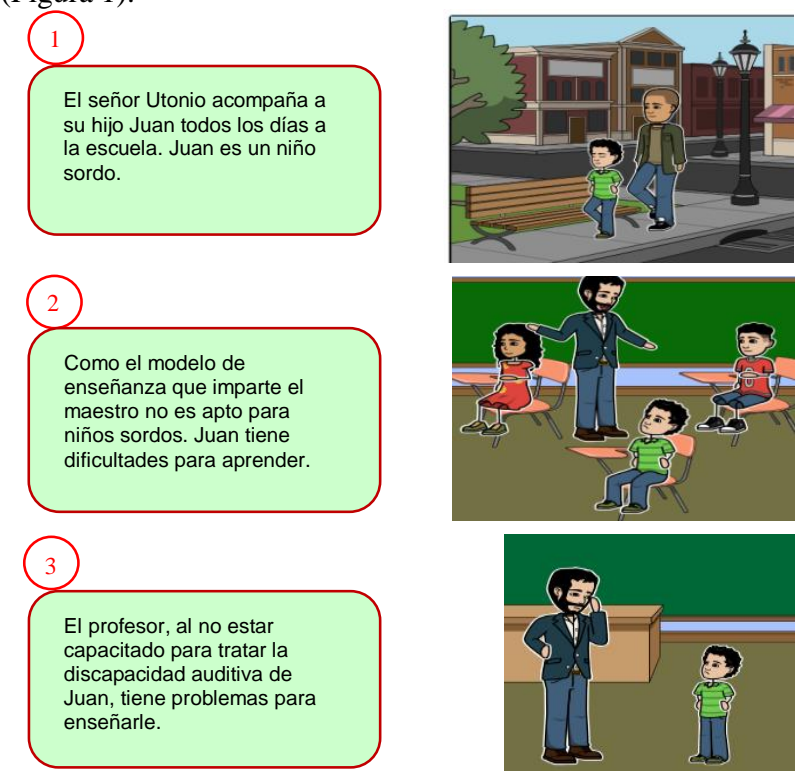

Figura 1. Storyboard: Niño sordo, dificultades de aprendizaje

Siguiendo al storyboard y las observaciones del estudio, se definió el perfil de usuario, que se muestra en la Tabla 1. El perfil de usuario se logró considerando la información de los niños y adultos sordos que participaron en el estudio. Se establece un rango de edad de cinco a 12 años, porque es donde presentan mayor dificultad en las primeras etapas de aprendizaje. Se considera el nivel preescolar debido a que a dicha edad están en constante aprendizaje y acuden a la primera institución educativa. También, cabe resaltar del estudio contextual, que los niños sordos desde pequeños deben desarrollar su memoria conceptual, lo cual se verá reflejado en mayor aprendizaje y capacidad de comunicación para sobrepasar las dificultades encontradas. 


\subsection{Diseño}

Para esta siguiente fase, se convierte en un reto proveer entonces la herramienta que pueda apoyar a los niños sordos en el contexto escolar. Se aprovecha la ventaja de que sí tienen cercanía con la tecnología. Además, se considera que las personas sordas a las que nos referimos pueden ver, por lo que esta característica será determinante.

Tabla 1. Descripción del perfil de usuario.

\begin{tabular}{|c|c|}
\hline Característica & Descripción \\
\hline Discapacidad & Sordera. \\
\hline Edad & Niños de entre 5 a 12 años. \\
\hline Nivel educativo & Cursar o haber cursado nivel preescolar. \\
\hline
\end{tabular}

De esta manera se da inicio al diseño de la aplicación móvil Tise-kuae Xuta Xtaya, que permitirá la mejora del aprendizaje de los niños sordos, ejercitando su memoria conceptual mientras aprenden el LSM. La aplicación propuesta, sigue dos de los principios de diseño de Donald Norman, contemplando el aspecto de visibilidad, incluyendo elementos gráficos y el de retroalimentación mediante una navegación guiada [7]. Nuevamente con apoyo de un storyboard, se representa el escenario en el contexto escolar con apoyo de la aplicación propuesta. El profesor tiene una herramienta de apoyo y puede dar un trato más directo al niño sordo, que puede hacer uso de lo aprendido, en este caso recordar y decir "hola" mediante el LSM a otra persona en algún otro lugar (Figura 2).

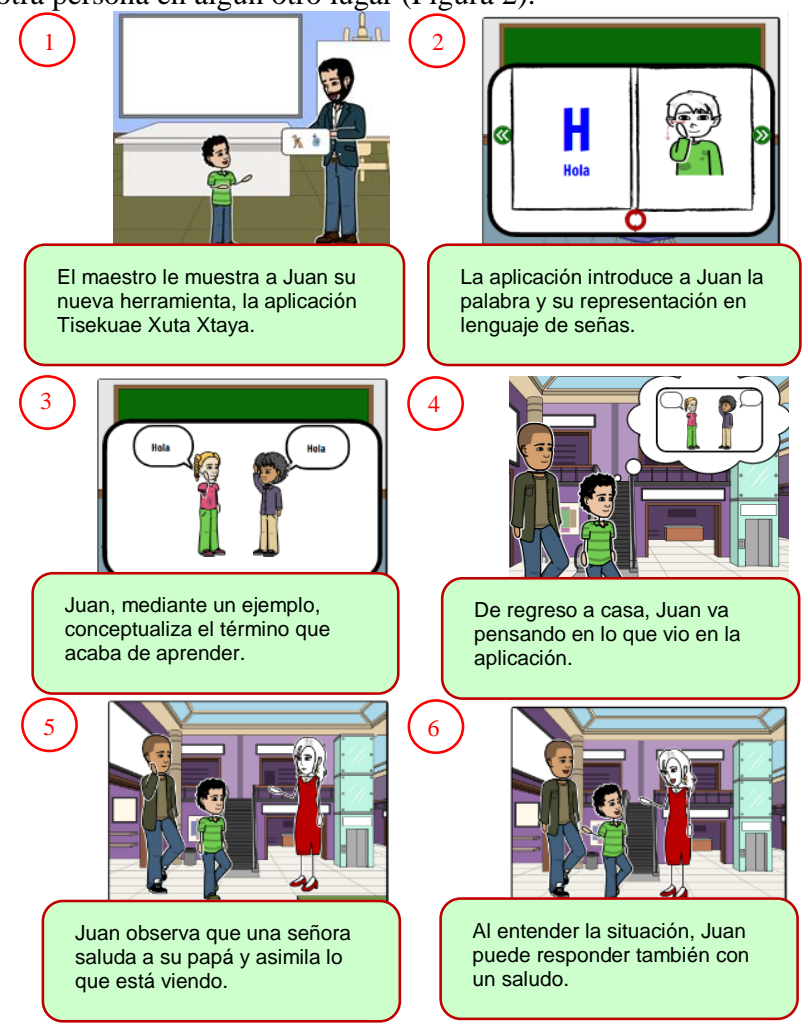

Figura 2. Storyboards: Niño sordo atendido con el uso de la aplicación TisekuaeXutaXtaya.
Después de haber realizado prototipos de papel, se define que la aplicación tendrá una interfaz de inicio y posteriormente un menú con diferentes temas de aprendizaje (Figura 3), basados en la Guía Deaf Child Worldwide [5].

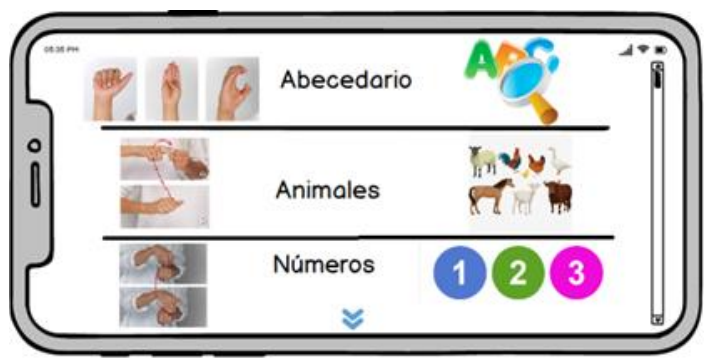

Figura 3. Interfaz de menú de Tisekuae Xuta Xtaya.

Al elegir una sección del menú, se mostrarán diferentes interfaces de aprendizaje (Figura 4) con siete elementos en común:

a. El nombre del objeto.

b. Representación gráfica del objeto (dibujo).

c. Una animación de la representación del objeto en LSM.

d. Botones de desplazamiento.

e. Botón para repetir animación.

f. Botón para observar animaciones de ejemplos.

g. Botón de inicio para regresar al menú.

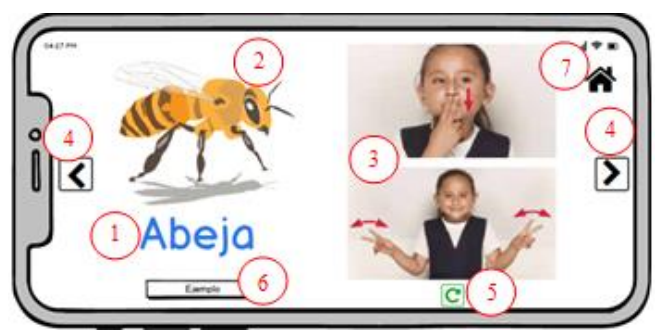

Figura 4. Interfaz de enseñanza.

Con los elementos presentados en esta interfaz, el niño podrá identificar el objeto en cuestión (2), saber mediante una animación, cómo se expresa en LSM (3), repetir la animación (5), y la manera en que se escribe (1). Las flechas laterales (4) permitirán regresar a la palabra anterior, o ir a la siguiente y podrá ver animaciones de ejemplo (6). Por último, el botón de inicio de la parte superior derecha (7) permitirá regresar al menú principal (Figura 3). Al apretar el botón (6) para ver más ejemplos en el repaso de la palabra, podrán conceptualizar lo aprendido, observarán una interfaz que ejemplificará el concepto revisado, como se observa en la Figura 5.

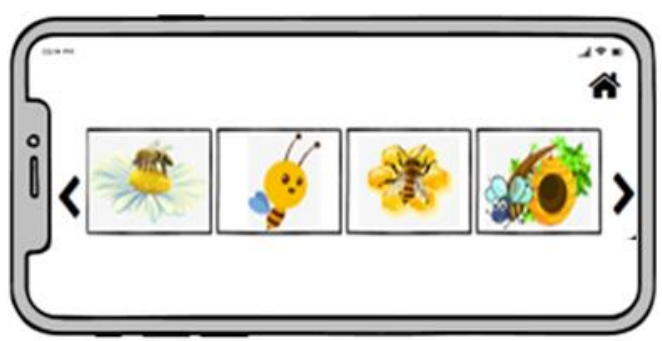

Figura 5. Interfaz de conceptualización.

Para el desarrollo de Tisekuae Xuta Xtaya, se realizaron dos versiones de prototipo. Se identifica del diseño de la aplicación a la interfaz menú, interfaz de enseñanza e interfaz de 
conceptualización. Hubo mejoras en las interfaces entre las dos versiones: en la interfaz de menú, se agregó también el nombre del tema en LSM; en la de enseñanza, se dio un mayor énfasis al objeto a enseñar y su representación en LSM, reorganizando los elementos; y en la interfaz de conceptualización, se agregó un mayor número de animaciones de ejemplo del elemento que el niño está aprendiendo, para lograr una mejor comprensión (Ver Figura 6).

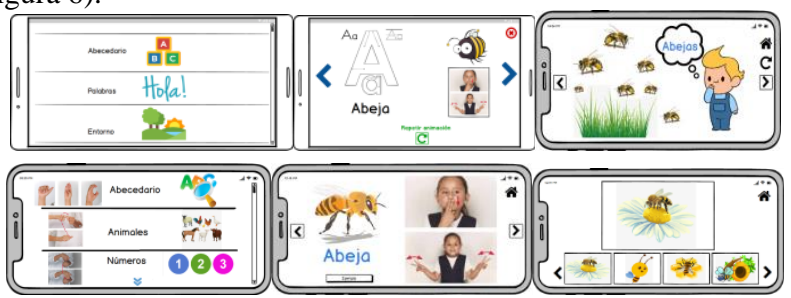

Figura 6. Aplicación Tisekuae Xuta Xtaya: Tisekuae Xuta Xtaya versión 1.0 (arriba); Tisekuae Xuta Xtaya versión 2.0 (abajo)

\subsection{Evaluación}

Se realizaron dos pruebas de Mago de Oz, la cual consistía en evaluar el prototipo in situ con base en capturas de pantalla (prototipos de papel) que dan la apariencia real de la aplicación. Los usuarios debían realizar las tareas: dirigirse al menú de los diferentes temas, visualizar el contenido de la interfaz de aprendizaje y repetir la animación de señas que ilustran las interfaces. Las funcionalidades eran simuladas por los involucrados en el proyecto, es decir, el cambio de una interfaz a otra de acuerdo a la acción realizada por el usuario, era mediante el cambio de capturas de pantalla. Los tutores fueron una parte fundamental durante las pruebas, pues ellos interpretaban las instrucciones que se daban. La primera prueba consiste en dirigirse al menú, y posteriormente en la segunda era elegir uno de los contenidos del menú, cada una de las tareas fueron realizadas por los usuarios con éxito, ya que los tutores comparaban los dispositivos móviles reales indicándoles a los niños la similitud con el prototipo, y puesto que los niños no son ajenos a estos dispositivos facilitaron las pruebas. La última prueba fue realizada con un poco más de dificultad, pues cuando el usuario veía la interfaz de aprendizaje (Figura 6, versión 1.0 imagen central) no entendía claramente lo que debía realizar por la sobrecarga de elementos en la interfaz (para todos los casos el usuario se la pasaba apuntando los elementos), la tarea se completó con éxito gracias al apoyo de los tutores, que instruían a los niños en lo que debían realizar (ver Figura 7).

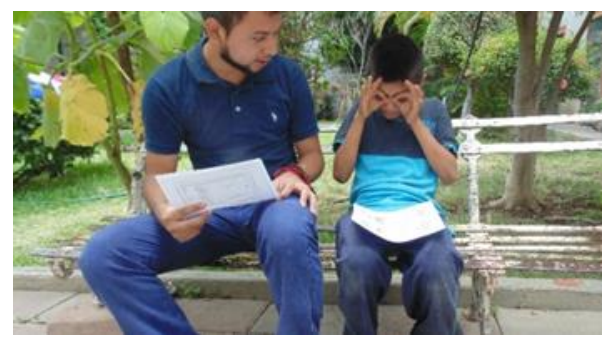

Figura 7. Pruebas de Mago de Oz.

Los resultados obtenidos de cada una de las pruebas son las indi-cadas en la Tabla 3. Si bien aún las pruebas de Mago de $\mathrm{Oz}$ no son pruebas de usabilidad de la aplicación funcionando en el móvil y sólo se aplicaron a dos usuarios, arroja un porcentaje de efectividad del $100 \%$ que es alentador e indica que vamos en la dirección correcta para ofrecer un producto usable. Las pruebas de Mago de Oz, permitió la realización de mejoras en el prototipo, las cuales se mencionan a continuación: Evitar la sobrecarga de elementos gráficos que puedan distraer y desorientar al usuario en el aprendizaje a corto plazo, sobre todo en la interfaz de aprendizaje (Figura 6, versión 1.0 imagen central). Y agregar una galería donde existan diversos ejemplos para fomentar la práctica de lo que está aprendiendo.

Tabla 3. Resultados de la prueba.

\begin{tabular}{|c|c|c|c|}
\hline $\begin{array}{c}\text { No. } \\
\text { Usuario }\end{array}$ & $\begin{array}{c}\text { Tareas } \\
\text { completadas }\end{array}$ & $\begin{array}{c}\text { Tareas no } \\
\text { completadas }\end{array}$ & $\begin{array}{c}\text { Tiempo (minutos) de } \\
\text { la prueba }\end{array}$ \\
\hline 1 & 3 & 0 & 12 \\
\hline 2 & 3 & 0 & 15 \\
\hline
\end{tabular}

\section{Conclusión}

La propuesta Tisekuae Xuta Xtaya, pretende ayudar en el contexto escolar y desarrollo de la memoria conceptual de los niños sordos. Con la ayuda de Tisekuae Xuta Xtaya, los niños sordos tendrán un acercamiento al uso de las tecnologías mientras aprenden diferentes cosas para comunicarse, de cara a su desarrollo en diferentes ámbitos. Si hay una continuidad en la atención de los niños como lo plantea la aplicación, se logrará hacer algo contra la barrera de la comunicación y el campo laboral en edades avanzadas, ya que los niños podrán comunicarse tanto en lenguaje español, como en lenguaje de señas con otras personas en sus mismas condiciones.

\section{Agradecimientos}

Se agradece a las personas de la comunidad de Teotitlán de Flores Magón por el apoyo brindado y disponibilidad para el estudio realizado, así como a las instituciones que nos brindaron información importante para el proyecto.

\section{Referencias}

[1] Organización Mundial de la Salud (OMS). Sordera y pérdida de la audición. http://www.who.int/es/newsroom/factsheets/detail/deafness-and-hearing-loss

[2] La discapacidad en México, datos al 2014. Instituto Nacional de Estadística y Geografía (México). 2016.

[3] Kimera (Colombia). Multimedia Kimera. 2006. http://www.kimera.com/

[4] Hablando con Julis (Colombia). Habla, lee y escribe con Hablando con Julis. 2005. https://www.hablandoconjulis.org/.

[5] Deaf Child Worldwide. Guía para los instructores de lengua de señas que enseñan a cuidadores, madres, padres y otros familiares de niños sordos. www.ndcs.org.uk/document.rm?id=8288

[6] Norman, D. y Draper, S. User Centered System Design: New Perspectives on Human-Computer Interaction. L. Erlbaum Associates Inc. Hillsdale, NJ, USA. 1986.

[7] Norman, D. A. The psychology of everyday things. New York: Basic Books. (1988) 